\title{
Heuristic Numerical Work in Some Problems of Hydrodynamics
}

\author{
By John R. Pasta and S. Ulam
}

$\mathrm{Jt}$ is well known that in many problems of hydrodynamics one cannot, at the present time, obtain valid solutions in a closed form. The asymptotic behavior of the solutions and even more generally their qualitative properties are barely obtainable through analytical work alone. This is even true of some problems which are already highly schematized by neglecting various physical parameters, like the change in the equation of state in a fluid or gas during its motion. One proceeds in numerical computations with the continuum of the fluid replaced by a finite network of discrete points, and ihus replaces the partial differential equations by a system of difference equations. The time variable, too, is replaced by a discrete succession of steps in time. This is the usual procedure in solving initial value problems which do not yield to analytical methods. In recent years the advent of electronic computing machines introduced the possibility of large scale experimentation in calculation of problems in more than one dimension.

It is the purpose of the following discussion to outline some general properties of such numerical work and to propose several different methods for numerical computations. Some numerical work already performed will be discussed in the sequel; it dates from 1952, when the authors first applied such computations on electronic computing machines. More recently, F. Harlow [1] has applied similar methods to calculations performed on electronic machines in Los Alamos. With the constant improvement in electronic computers, both as regards their speed and the size of the memory, it will be possible to perform more ambitious calculations; both the variety and the magnitude of the problems which can be handled will increase. Such calculations can play a role analogous to that of experiments in physics and may suggest new theoretical lines of attack.

There are, broadly speaking, at least two different ways to approach numerically the problems dealing with the dynamical behavior of continua. The kinetic theory of gases assumes the physical reality of the discontinuum. One calculates the properties in the large of the motion of $N$ "points"-atoms or molecules which have statistically given velocity distributions and are subject to incessant collisions with each other. There are forces acting between these points, e.g., deriving from potentials, but whose form is only imperfectly known. Many, but not all, properties of the macroscopic motions are largely independent of the exact form of the interactions between these particles. This is not the place to enter into a description of the way to derive the hydrodynamical equations from the Boltzmann integral-differential equations describing the microscopic behavior of such systems. Suffice it to say that, e.g., Navier-Stokes equations can be so derived and they will describe the behavior of certain statistical averages (or functionals on the $6 N$ dimensional space) which are interpreted as macroscopic quantities like density, pressure and velocity, of a point in space (three dimensions) as functions of time.

Received $12 \mathrm{May}$, 1958. This work was performed under the auspices of the U.S Atomic Energy Commission. 
What we prefer to discuss here briefly is the numerical procedures which are suggested by this model. The number $N$ is, in problems of hydrodynamics, enormous. $N$ is greater than, say, $10^{22}$ for 1 cc of liquid. Is it possible to scale down this number to a value practical for numerical computations and still be able to observe meaningfully the behavior of the functionals in which we are interested?

The answer, it seems to us, is in the negative at the present time. If one takes the Boltzmann equations literally and considers the individual points of calculation as representing atoms, then to obtain the "average" velocity of a point of the gas one would require, say, $k$ particles per cell in a spatial resolution in which we are interested. Let the number of cells be $l$. (In practice, the resolution equals $d$ on a linear scale and $l$ equals $d^{r}$; where $r$ is the number of dimensions, and equals 1, 2, 3 (in problems in one, two, or three dimensions without special symmetries reducing the number of independent space parameters).) The total number of points is then $\vartheta=k d^{r}$. If we want a statistical error of the order of $1 \%$, then $k \sim 10^{4}$. If the linear resolution is to be of the order of $5 \%$, say, then $d$ is 20 . Even in one dimension, we would have to consider 200,000 points. This is much too high for present computers and should make it clear that in a numerical calculation, the "points" have to be thought of as representing not individual atoms but rather large aggregates of atoms. The behavior of each point has to be schematized so as to represent a statistical average of a great number of atoms. The numerical work will not reflect the Boltzmann equations, but the simpler equations which are its consequences; each point of our calculation represents, for example, the center of mass of a collection of atoms. The implicit assumption in this set-up is, therefore, that such a mass remains coherent during the entire course of the problem. That is to say, the molecules initially close to each other remain so throughout the problem and do not diffuse too much with respect to each other-the small globule of the fluid does not distort too much.

Parenthetically, we may add that this question is connected with the whole complex of difficulties which one encounters even in the purely mathematical studies in the theory of functions of several real variables. The so-called density theorems and the notion of set-derivation can serve as examples: The density of a set of points at a given point has to be defined through a sequence of sets such that not merely their diameters shrink to zero, but the sets have to be "not too thin"-for example, in case of rectangles enclosing the given point, the ratio of the sides has to remain bounded. This sort of assumption is necessary for the validity of Vitali's theorem, etc. Physically it is clear that in our case we have to assume that the surface-tovolume ratio for the globules has to remain bounded for any a priori evaluation of the pressure, which acts on its center of mass. One has to introduce pressure, and there seem to be at least two obvious ways to do it:

a) Assuming the knowledge of the equation of state, there is the dependence of the pressure on local density (we assume as given the thermodynamic nature of the process). In this outline we may specialize, say, to either an isothermal or adiabatic process, that is, $p=f(\rho)$. The pressure gradients which are evaluable through gradients of density will be calculated then by estimating these gradients through the geometry of the instantaneous appearance of our system of points which represent, we emphasize, the positions of centers of mass of globules. Our problem, then is that of finding a rule or recipe to estimate the density at a point of space given 
only a finite system of points (rather widely separated!). The limiting case of an enormous number of points presents no difficulties, for one could simply count the number of points in a square of a fixed mesh, and this number will be proportional to the density. In practice, since we are limited to a moderate number of points for the whole fluid, the question is to estimate the density in the most reliable way. Consider the case of two dimensions. We can think of the points located initially in a regular array, for example, on the vertices of a rectangular division, or better, points of a triangular subdivision of space. Of course, after some "cycles" of the computation, the geometry of the system will change and one could proceed to estimate the density as follows: Enclose each point by triangles with the closest points as vertices. The smallest (in area) triangles in whose interior a given point is located would give, through the ratio of its area to the area of the original triangle, at least an idea of the change in density at that point.

This procedure suffers from several drawbacks. There is the question of the computational stability of the calculation. The selection of the nearest points leads to discontinuity, in time, of the area. In a rectangular subdivision the more "classical" definition of density through the Jacobian in a finite approximation requires the knowledge of the position of four nearby points $x \pm h, y \pm k$. In the equations of motion for each of our points, we need the gradients of the density in the Lagrangian coordinates (the " $a, b, c$ " not the " $x, y, z$ "). In our crude way of calculating the densities themselves, the computation of differences in fixed directions in space at a given time, the nearby points for increasing $a, b, c$ may not be sufficiently close, and the gradients may be very inaccurately estimated. In the case of points in the boundary of the fluid, say with vacuum, one needs special prescriptions. All this introduces even more serious errors.

b) There is another way to introduce, numerically, the forces due to pressure gradients. We could imagine repulsive forces acting between any pair of our points. They would in simple cases depend on the distance alone (the forces should derive from potentials if we assume scalar pressure-no tensor forces-no viscosity, etc.). The form of the potential will, of course, depend on the equation of state. In a onedimensional problem the nature of this correspondence is clear-it suffices to have forces between neighboring points only. The continuity of motion guarantees the permanence, in time, of the relation of neighborhood. The situation is however completely different in two or more space dimensions. (A general remark here: In one-dimensional problems the points of our calculation can best represent the boundaries of zones into which our fluid or gas is sub-divided. These keep their coherence and shape, and the only meaningful parameter for the spatial distribution, the density, is inversely proportional to the width of the zones. In two or more dimensions the boundaries of zones are not easily describable by points or systems of a few points, and these points, we will repeat once more, correspond to centers of mass of volume.) We have to define the neighbors of a given point. One can do it in reference to the original geometry of a system of points that is to keep the relation which existed initially. But this is not good except in the case of infinitesimal or small deformation. The neighbors of a given point will change in the course of time. If one tries to calculate the resultant force on a point by calculating it from all the points in the mesh, not merely the neighbors, one should remember that the number of calculations increases with the square of the number of points. A "cut- 
off" for the force is necessary at some distance and the force is not calculated if the distance between two points exceeds a certain constant. One has to remember that in the initial position, that is to say, in the regular lattice in which the points are arrayed, the cut-off has to be selected so that with it we obtain the initial distribution of pressure. The pressure gradient is given directly as the resultant of all the forces acting on a point from its "neighbors" and depends on the actual position of the point without reference to the ("forgotten" by the system) initial configuration. We will discuss in the sequel, for some concrete problems, how such calculating schemata operated.

To summarize briefly the above computational scheme: The particles represent small parts of the fluid. The forces due to pressure gradients are introduced directly by imagining that neighboring or "close" points repel each other. The dependence of this force on the distance between points is so chosen that in the limiting case of very many points, it would represent correctly the equation of state. That this is possible, in principle, is clear a priori: the density is inversely proportional in the limit of a very large number of points to the square (in two dimensions) or cube (in three dimensions) of the average distance between them. The pressure is a function of density, and this being a function of the distances, we obtain an analogue of the equation of state by choosing a suitable distance dependence of the force. The Lagrangian particles are at $P_{1}:\left(x_{1}, y_{1}, z_{1}\right), P_{2}:\left(x_{2}, y_{2}, z_{2}\right), \cdots, P_{N}$ : $\left(x_{N}, y_{N}, z_{N}\right)$. The forces (repulsive) between any two are given by $F_{i, j}=$ $F\left(d\left(P_{i}, P_{j}\right)\right)$ where $d\left(P_{i}, P_{j}\right)$ is the distance between the two points.

The average value of $d$ at a point of the fluid is, in the limit of $N \rightarrow \infty$, a function of the local density: $\bar{d} \simeq \rho^{-1 / 3}$.

The pressure $p$ is a function of $\rho$ alone in, say, isothermal or adiabatic problems. The pressure gradients give then the expression for our $F$.

There is so far no general theory and the convergence of such finite approximations to the hydrodynamical equations remains to be proved, but even more important than that would be an estimate of the speed of convergence.

One could assume, as a starting point for a numerical calculation, instead of the partial differential equations of hydrodynamics, e.g., the equations of Euler-Lagrange, a mathematical description which is somewhat more general: the "points" of our calculation need not correspond to the material points of a fluid, but instead may represent-more generally-some other parameters of the problem.

After all, in many problems one is not interested in the positions of every given particle of the fluid, but rather in the behavior, in time, of a few functionals of the motion; for example, if $a, b, c$ are the "laboratory" or Lagrangian coordinates in fixed space in the classical formulation, one deals with the functions $x(a, b, c, t)$, $y(a, b, c, t), z(a, b, c, t)$ which are interpreted as a position at time $t$ of the point which at time $t=0$ occupies the point $(a, b, c) . \rho(x, y, z, t)$ is the local density which is computed by differentiation from the knowledge of $x, y, z$ and the derivatives

$$
\frac{\partial x}{\partial a}, \frac{\partial x}{\partial b}, \frac{\partial x}{\partial c}, \frac{\partial y}{\partial a} \cdots \text { etc. }
$$

The pressure $p$ is, for our purpose, computable from $\rho$. It is always possible to think of the functions $x, y, z$ (which satisfy the Euler-Lagrange equations) as developed into series: 


$$
\begin{aligned}
& x=\sum_{i, j, k} \alpha_{i j k}(t) \psi_{i j k}(a, b, c) \quad z=\sum_{i, j, k} \gamma_{i j k}(t) \psi_{i j k}(a, b, c) \\
& y=\sum_{i, j, k} \beta_{i j k}(t) \psi_{i j k}(a, b, c) \\
& i, j, k=1,2, \cdots
\end{aligned}
$$

Here the $\psi$ 's are fixed functions of $a, b, c$ alone. The functions need not form orthogonal systems but, to fix the ideas, we might think of them as being terms of the Fourier series of Rademacher functions, for instance. The $\alpha, \beta, \gamma$ are functions of time alone and can be treated as abstract Lagrangian particles. The partial differential equations for $x, y, z$ are replaced by a system of infinitely many (a discrete infinity) of ordinary differential equations describing the change in time of the $\alpha, \beta, \gamma$. To see the validity of such an approach, we shall illustrate this proposal in an example.

In a one-dimensional problem, we have to find the function $x(a, t)$ satisfying the equation

$$
\ddot{x}=-\frac{\partial p}{\partial x}-F(x) ; \quad p=f(\rho) ; \quad \rho=\frac{\rho_{0}}{\frac{\partial x}{\partial a}}
$$

with a given initial distribution of density and velocity of the fluid. The ordinary numerical procedure for a solution consists in the replacement of the continuous variable $x$ by a discrete one, that is, $x(a)$ is replaced by $x_{i} \quad i=1,2, \cdots N$. Each $x_{i}$ obeys a Newtonian equation of the second order for the $x_{i}(t)$ where in practice we also replace $t$ by a discrete sequence of times and obtain a system of difference equations. Our introduction of the fixed functions of space $\psi(x)$ and the $\alpha_{i}$ 's amounts to a Lagrangian change of variables where instead of the $x_{i}$ which are the actual "points" of our fluid, we introduce new variables $\left(q_{i}\right)$ in Lagrangian notation:

$$
q_{1}=q_{1}\left(x_{1} \cdots x_{n}\right), \quad q_{2}=q_{2}\left(x_{1} \cdots x_{n}\right) \cdots q_{k}=q_{k}\left(x_{1} \cdots x_{n}\right)
$$

where the functions are "holonomic", that is, they do not involve the velocities $\dot{x}_{i}$ in a non-integrable form. For example, the analogue of the Rademacher functions would be: If $n$ is of the form $n=2^{k}$

$$
\begin{aligned}
q_{1} & =\sum_{i=1}^{n / 2} x_{i}-\sum_{i=(n / 2)+1}^{n} x_{i} \\
q_{2} & =\sum_{i=1}^{n / 4} x_{i}-\sum_{i=(n / 4)+1}^{n / 2} x_{i}+\sum_{i=(n / 2)+1}^{(3 / 4) n} x_{i}-\sum_{i=(3 / 4) n+1}^{n} x_{i}
\end{aligned}
$$

The differential equations for the $x_{i}$ will be replaced by a system of equations for the $q_{i}$; the forces which are given directly for the $x$ variable by pressure gradients will be replaced by "generalized" forces which are functions of the $q_{i}$ and their derivatives. In cases where the $\psi$ form an orthogonal system, the kinetic energy will still be a quadratic function of $q_{i}$ and the $\alpha_{i}$. This procedure is, of course, strictly legitimate in the case where we consider the $x$ variables being expressible by the $q_{i}$.

A general question arises: Which, in a given problem, are the most convenient variables $(q)$ ? It is clear that in many problems of hydrodynamics one is interested 
mostly in certain over-all properties of the motion of the fluid-one wants to know the behavior of certain given functionals.

In the classical approach one calculates first the $x, y, z$ for each of the points of the space $a, b, c$. In practice, one is limited to a finite number of these points, and it is perhaps plausible that in many cases it would be "better" to know, instead, an equal number of coefficients $\alpha_{i j k}$ in the development of $x(a, b, c, t)$ in a given (Fourier) series than to know the value of $x$ in a corresponding number of points in the space of the $\alpha_{i j k}$ 's. This is especially clear if one should know, a priori, from the physical nature of the problem that the functions $x, y, z$ are reasonably smooth (e.g., in the sense that the absolute value of the partial derivatives remains bounded). In the terminology of the $\alpha_{i j k}$ 's, this smoothness amounts to the knowledge that the coupling between the $\alpha$ 's which have high indices and those with small values is small. That is to say, the series converge rapidly. Physically speaking, it means that the high frequencies are less excited.

Such reduction of a continuum to a discrete countable infinity is, of course, very familiar in some problems of quantum theory - the radiation field, etc. The problems that have been dealt with in quantum theory have been mostly linear; there are no forces between the coefficients and each mode or "particle" represented by the coefficients behaves independently of all the others.

For the case of our hydrodynamical problems, the forces due to pressure gradient are functions of all the coefficients; that is, we have a true $n$-body problem, and the "quantization" is justified practically only if a cut-off at a finite index $(i)$ is permissible together with an estimate, in advance, of the error for all $t$ under consideration-that is to say, if the high modes do not become increasingly important. When the high modes do acquire more energy as time goes on, the classical approach becomes difficult also. The onset of turbulence or of the positional mixing of the fluid renders the classical treatment (partial differential equations) illusory. The absolute values of spatial derivatives increase and even their existence for finite times cannot be guaranteed and a statistical approach is indicated. It would seem that in problems where this behavior is expected, the approach through a study of a finite model involving perhaps a change of coordinates, like the one proposed, may be of possible utility. If one wants to study the rate of development of instabilities or the rate at which mixing proceeds, then the flow of energy from the low modes (that is to say, from the $\alpha$ 's with small indices to those with high indices) will show just the rate of increase in mixing positionally or, in the derivatives, the rate of increase in vorticity.

We would like to mention another possible advantage in the use of general coordinates in numerical work. A description of the motion of the fluid through the partial differential equations of hydrodynamics postulates the existence of the partial differential expressions. It is well known that even in comparatively simple problems these derivatives exist only up to a finite value of time, after which discontinuities develop, e.g., in pressure and in density (that is to say, shocks), and one has to use different methods to treat such discontinuities. On the other hand, a Rademacher series or Fourier series may very well represent a step function, more generally functions without derivatives at some points. The $\alpha_{i}$ might then continue to be used as dynamical variables even after discontinuities occur in de- 


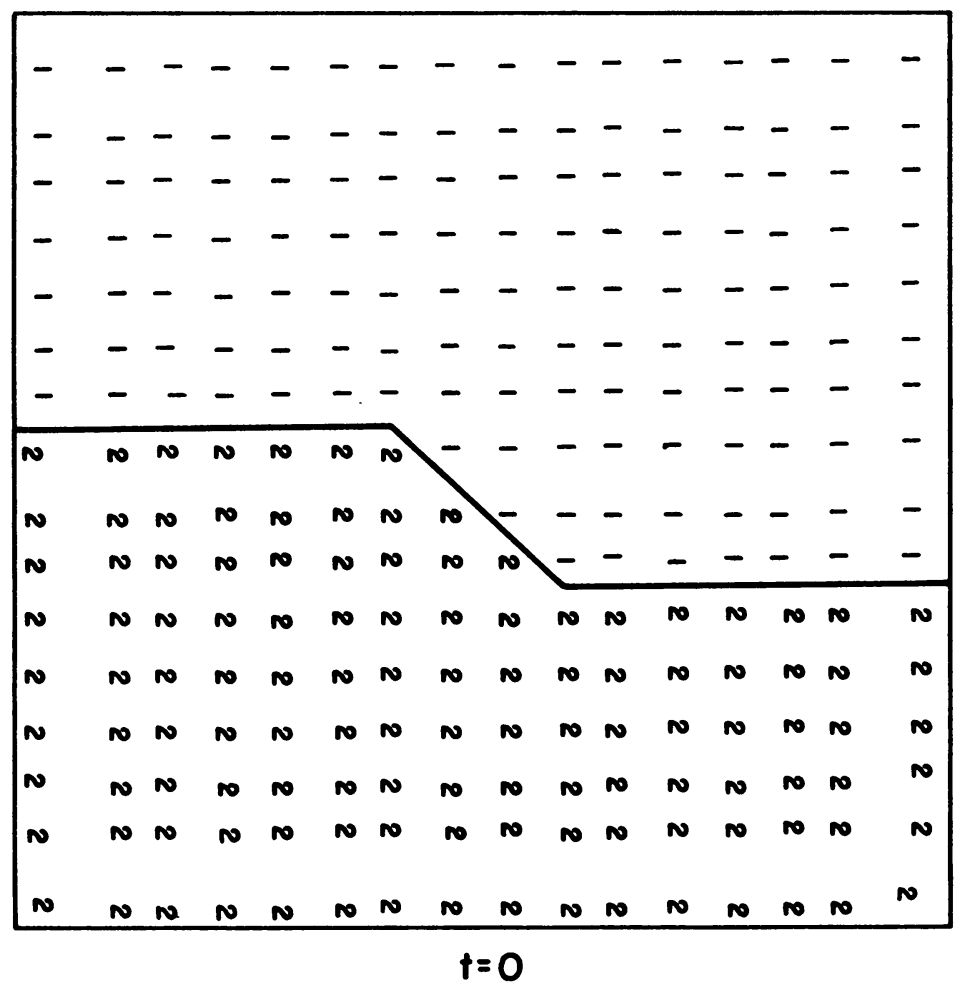

FIg. 1.-Initial configuration, $t=0$

rivatives like $\partial x / \partial a$ making the differential expressions in the Euler-Lagrange equations unmanageable.

In order to test some of these general speculations on actual problems, we have run some numerical computations on an electronic machine, the MANIAC, in the Los Alamos Scientific Laboratory and on its prototype at the Institute for Advanced Study in Princeton. The main purpose of these calculations was exploratory, and the feasibility of using certain numerical schemes on the machine was considered of more interest than the precision of the results. The main point of interest was the amount of time necessary in order to compute on the machine the time behavior of certain functionals of our systems. The problems were mostly of the initial value type, the integration was in time, and the calculation ran in cycles, for which we decided that about five minutes would be allowed. The nature of the problems and the characteristics of the machine with this requirement fixed the maximum number of mass points at about 256. The first problem studied involved the motion of a heavy fluid on top of a lighter one--usually known as the Taylor instability configuration.

The initial configuration was the $16 \times 16$ array shown in Fig. 1 . The particles are of two types, one having a mass which was double the mass of the other. The force was the same between any two particles and was chosen to be inversely proportional to the distance of separation. This algebraically simple choice was made 
mainly to keep the computation time to a minimum. The whole system of particles is enclosed in a unit square with the heavier particles on top and all particles subject to an external gravitational field, an unstable configuration. With our simple force law the computation for each pair of particles took about 30 milliseconds. With our 256 particles, or over 32,000 pairs, the computation of resultant forces would have taken 15 minutes. This time can be reduced by introducing a cut-off in the force so that for a particle lying outside such range of influence, the only computation necessary is that of a separation between the particles. In order to avoid even such computations of distance which involve the long multiplication time of the machine, we arranged the cut-off in terms not of the Euclidean metric, but in terms of another one (Minkowski): the sum of the horizontal and vertical distances of separation thereby reducing the time of ascertaining the distance in such case to about 3 milliseconds for these pairs. With this, the total computation time (for a cycle) was about 5 minutes, and a typical problem would run 150 cycles -all together, with printing of results, somewhat more than 10 hours.

When a particle approached the side of the container closer than a cut-off distance, a special situation arose. The most convenient way of treating the wall was to create a virtual particle located on the wall at the same horizontal or vertical position as required to contain the real particle. Again we should emphasize that for our first experiments, this recipe was chosen for computational conveniences rather than as a mathematical or physical requirement. Thus, also, all the parameters for the problem were chosen to be simple powers of 2 so as to be able to use the fast operation of the left and right binary shift, rather than the slower operation of direct multiplication.

With our grid of points, crudely as it was chosen, it hardly can be expected that the details of the motion will be exactly described. A typical configuration is shown in Fig. 2; a much later cycle (later time) configuration is shown in Fig. 3 and the formation of the "atmosphere" is apparent. What can be hoped, however, is that some functionals of the motion will be more accurately depicted. We are interested in the transition phase and in the time rates of mixing on a large scale between the two fluids. One of the functionals which we observed and plotted as a function of time is the total kinetic energy of the particles divided into two parts: the kinetic energy of the horizontal and vertical motions separately (these are shown on Fig. 4 ). Even though the motion itself is very irregular, these quantities seem to present rather smooth functions of time. In the case of a stable configuration with the lighter fluid on top and the heavy fluid on the bottom, there would be only a periodic interchange of kinetic and potential energies.* In the unstable case, there should be an increase of kinetic energy which persists for a considerable time. The hope is that with many more calculations one could try to guess from such numerical results at least the form of an empirical law for the increase of this quantity as a function of initial parameters.

Another quantity of interest is the spectrum of angular momentum which may be defined, for example, in the following way: we draw around each particle a circle of fixed radius and calculate the angular momentum in each such region. We then

\footnotetext{
* See Fig. 5, showing the configuration at a time later than the one in Fig. 2.
} 


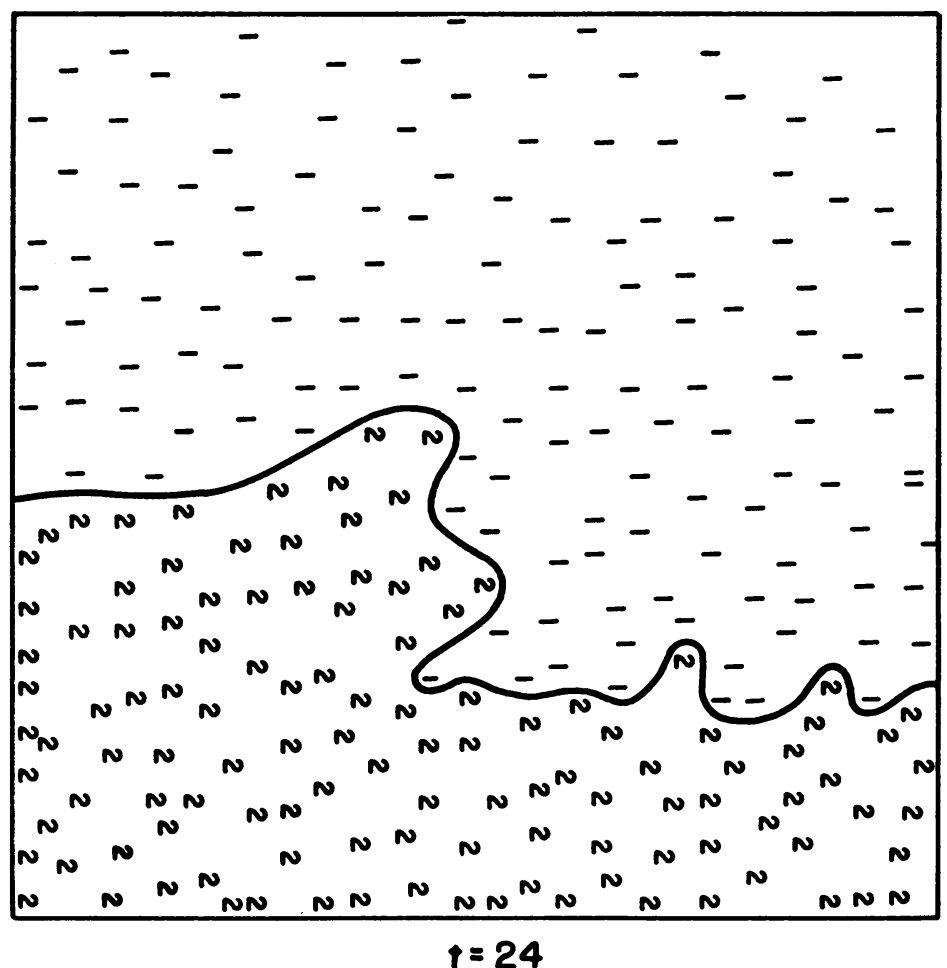

FIg. 2.-Typical configuration, $t=24$

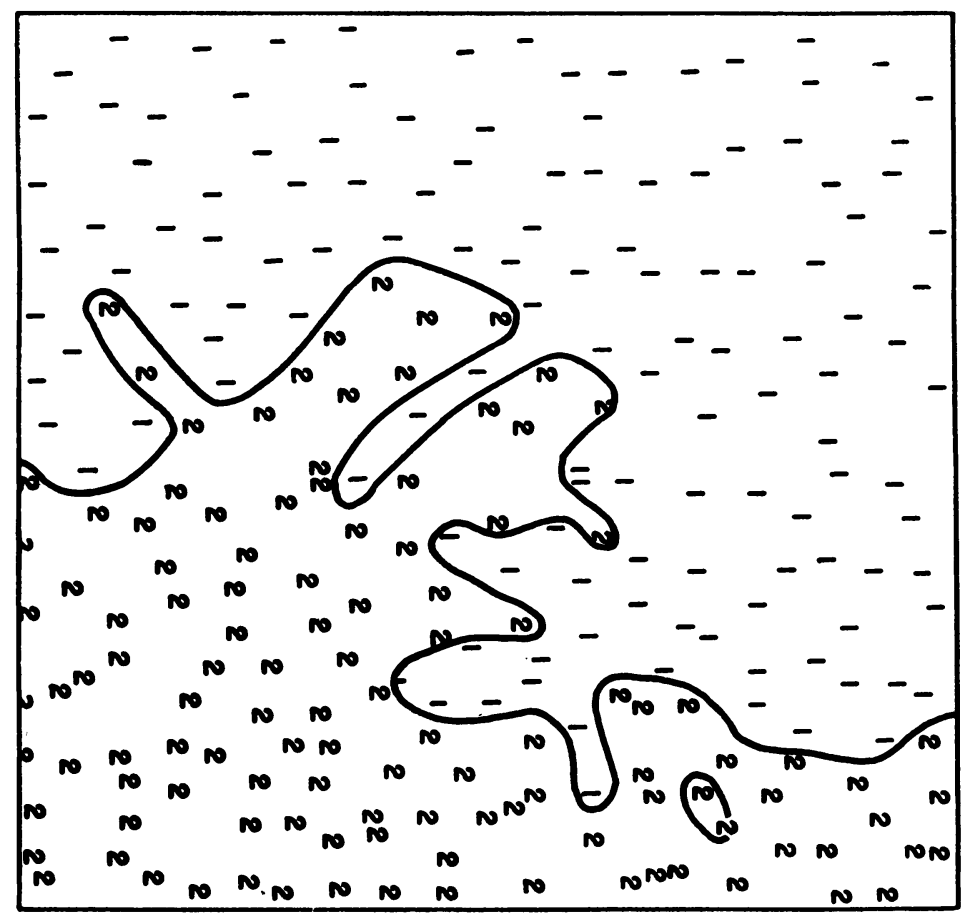

$$
t=46
$$

FIG. 3.-Typical configuration, $t=46$ 


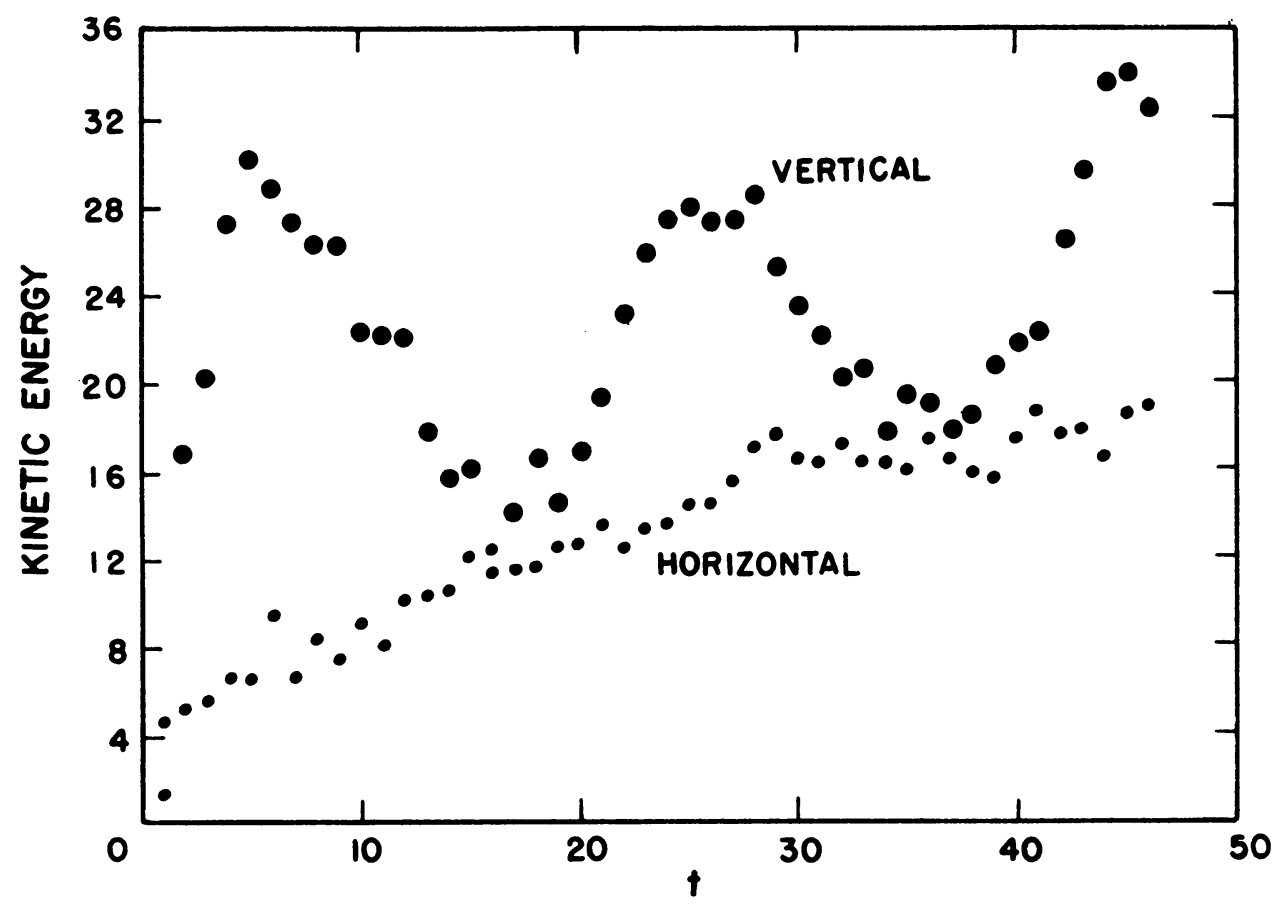

FIg. 4.-Kinetic energies of horizontal and vertical motions

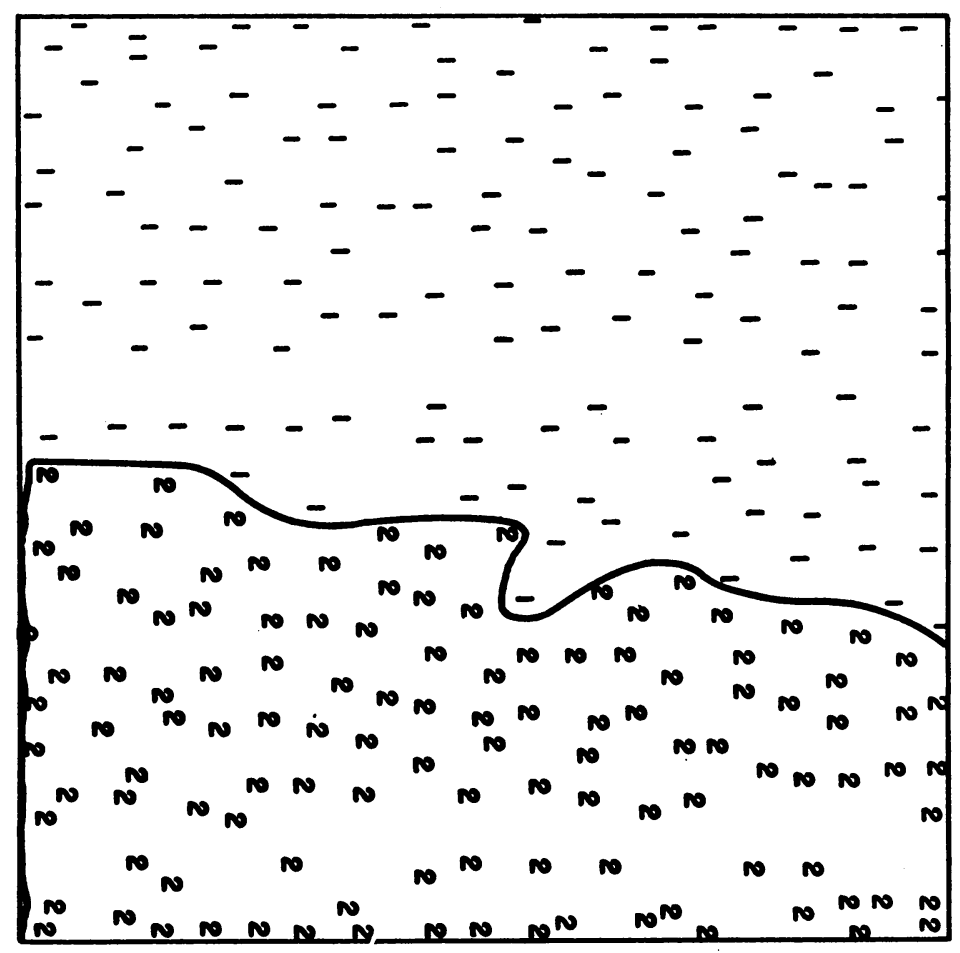

STABLE CASE

Fig. 5.-Typical configuration, stable case 
take the sum of the absolute values or the sum of the squares of these quantities. This gives us an over-all measure of angular momentum on a scale given by the radius. Varying the radius, these numbers may then be studied as a function of both time and the radius. It is interesting to consider the rate at which the large scale angular momentum is transferred to small scale eddies-also, the spectrum in the asymptotic state, if it exists. All this, of course, as a function of the parameters of the problem, the ratio of the densities of the two fluids and the external force. It is clear that very many numerical experiments would have to be performed before one would trust such pragmatic "laws" for the time behavior of mixing or increase in vorticity.

Another reason for selection of our problem was an interest in the degree of spatial mixing of two fluids, starting from an unstable equilibrium. To measure quantitatively such mixing in configuration space, a functional was adopted similar to the one just mentioned for the spectrum of angular momentum. At a given time $t$, a circle is drawn around each point $P$. In each circle, we look at the ratio $r$ of the number of heavy particles to the total number of particles in this region. We take the quantity $\mu(P, t)=4 r(1-r)$. This gives an index of mixing of the fluid in the circle, being equal to zero if only one fluid is present and equal to one if particles of both fluids are equally present in it. We average this quantity over all the circles. Initially this average is very close to zero, the only region where it differs from zero being around the interface. As the mixing proceeds, our average measure of mixing increases with time. We have plotted this quantity for several different radii. These functions are again comparatively smooth even though the interface between the two fluids becomes highly convoluted. Again, one might expect that after a sufficient number of numerical experiments, one would obtain a hint of the time behavior of this "mixing functional", or at least an idea of the time $T$ taken for the over-all mixing (which is initially close to zero) to become of the order of $\frac{1}{2}$ or $1 / e$, say. This time, for dimensional reasons, must depend on $\sqrt{L / a}$ where $L$ is the depth of the vessel and $a$ the acceleration of the lighter fluid into the heavier one.

The problem of Taylor's instability in its initial stages was also examined in Los Alamos experimentally for the case of a heavy gas on top of a light gas. The interface between the two gases involved an irregularity. Photographs taken at various times show configuration of the two gases not unlike those developing in our calculation. If one wanted to put credence in calculations like the above, the form of the proper force law imitating the real equation of state would have to be chosen very carefully. Also one would have to study the question of how the accumulation of error due to the finiteness of our grid will effect the behavior of our functionals. We have treated compressible gases. An attempt to treat numerically incompressible fluids in a similar way encounters serious additional difficulties:

In order to preserve the volume of each fluid element, one would have to keep constant the areas, in two-dimensional problems, of triangles or other elementary figures whose vertices are occupied by our particles. This means that there is a great number of constraints added to the equations of motion for the repelling mass points. This was computationally not feasible on the available machines for problems where the number of points was of the order of 100 or more. 
Another way of calculating in the above spirit would have been to postulate additional forces, doing no work but strongly tending to preserve the elementary area defined by our mass points. This also turned out to be impractical.

Atomic Energy Commission, Washington, D. C. and

Los Alamos Scientific Laboratory, Los Alamos, New Mexico

1. M. W. Evans \& F. H. Harlow, The Particle-in:Cell Method for Hydrodynamic Calculations, Los Alamos Report 2139, 1957. 